\title{
Transmitted light microscopy of a fibre reinforced metal
}

\author{
B. MOSER*†, A. ROSSOLL*, L. WEBER*, O. BEFFORT $\dagger \&$ \\ A. MORTENSEN* \\ *Laboratory for Mechanical Metallurgy, Swiss Federal Institute of Technology EPFL, Lausanne, Switzerland \\ †Section Materials Technology, Swiss Federal Laboratories for Materials Testing and Research, EMPA, \\ Thun, Switzerland
}

Key words. Alumina fibre reinforced aluminium, fibre fracture, fibre reinforcement, metal matrix composites, metallography, transmitted light microscopy.

\section{Summary}

A method is presented for studying fibre damage in continuous fibre reinforced composites. It is based on contrasting the transmission of light through intact translucent fibres with the light through fractured or dead-ended fibres. The method is applied in order to detect processing-induced fibre fractures in aluminium reinforced with continuous alumina fibres.

\section{Introduction}

Broken and dead-ended fibres in fibre-reinforced composites can cause stress concentrations and thus trigger fibre breakage in adjacent fibres and cause final fracture of the composite. Knowledge of where and how many of these inhomogeneities are present is key for understanding composite fracture.

When a ceramic fibre reinforced metal is prepared for metallography and observed in the reflected light microscope with the fibres orientated perpendicular to the polishing plane, the fibres appear as dark circles against the light-reflecting metal matrix. Some fibres, however, appear far lighter than the rest; one such fibre is visible in Fig. 1. Clearly, in these fibres the incident light is reflected from some defect beneath the surface. The nature of this defect is not identifiable directly because it is situated too far from the polished sample surface to be examined under the light microscope.

In the present investigation, we propose a method that allows the examination of such defects in fibre composites and we positively identify one type of defect. The method is based on the use of transmitted light microscopy. We show that this technique, although relatively seldom used in the study of

Correspondence: B. Moser, LEXCOM, Massachusetts Institute of Technology MIT, Cambridge, MA, USA. Tel.: +1 617253 9825; fax: +1 617258 0390; e-mail: benedikt.moser@alumni.ethz.ch structural inorganic materials, can offer an interesting tool for the characterization of continuous fibre reinforced composites.

\section{Materials and experimental procedures}

The pure aluminium matrix composite wire studied here is reinforced with 50 vol. $\%$ Nextel $610^{\mathrm{TM}}$ continuous alumina fibres. It is produced commercially by the $3 \mathrm{M}$ company (St Paul, MN, USA) using a continuous ultrasound-driven liquid metal infiltration technique in which the fibre tows are pulled through a bath of the molten metal as described in McCullough et al.

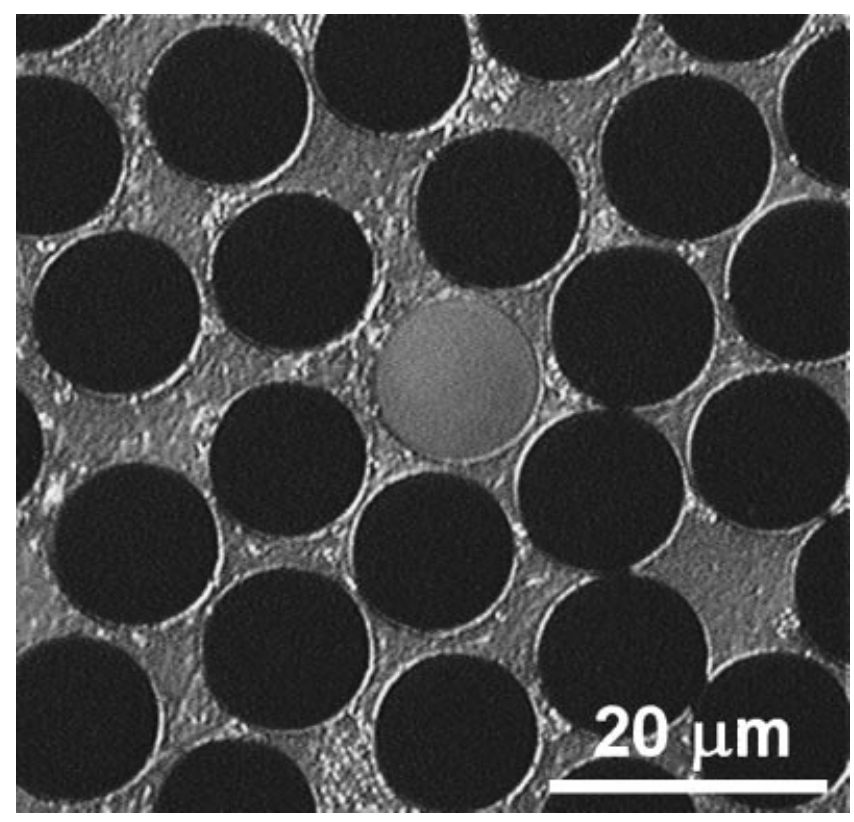

Fig. 1. Optical micrograph in the reflected light mode. In the case of the bright fibre, the incident light is reflected on some defect beneath the polished surface. 
(2001). An ultrasound horn is immersed in the matrix bath and drives the infiltration. The properties of the continuous $\alpha$-alumina fibre can be found in Bunsell \& Berger (2000), Wilson \& Visser (2001) and 3M Company (2001).

The composite wire has a Young's modulus of about $220 \mathrm{GPa}$ and a strength exceeding $1300 \mathrm{MPa}$. Its electrical conductivity is higher, whereas the coefficient of thermal expansion and density are lower, than values for stainless steel; this has driven its development as a core material for overhead power transmission lines (3M Company, 2002). The wire has a circular cross-section with an equivalent diameter of roughly $2 \mathrm{~mm}$.

\section{Metallographic procedure}

In preparation for transmitted light microscopy, the specimens are metallographically polished on both sides. For this purpose the composite wire is glued with a filled single component epoxy glue (Scotch Weld 2144, 3M) in a steel tube (inner diameter $3 \mathrm{~mm}$, outer diameter $6 \mathrm{~mm}$ ), and slices of different thicknesses are cut perpendicular to the fibre direction. Cyanoacrylate rapid glue serves to glue these slices on a sample holder that is designed to keep several specimens with different thicknesses on the same height. The specimens are then mechanically polished on an automatic polishing system Phoenix $4000 \mathrm{~V}$ (Buehler GmbH, Düsseldorf, Germany) using a standard diamond polishing procedure (Moser, 2002). After polishing the first side, the rapid glue is dissolved by immersing the whole specimen holder in acetone, the specimens are glued upside down and the reverse side is polished. Then the glue is dissolved again and the specimens are cleaned.

\section{Microscopy and quantitative metallography}

An Axioplan 2 optical microscope (Carl Zeiss, Oberkochen, Germany) with two $100 \mathrm{~W}$ halogen light sources (one for the transmitted and one for the incident light beam) equipped with a CCD camera was used to take digital micrographs. The power of the transmitted light beam was adjusted depending on the specimen thickness. A slight amount of incident light was always used at the same time to illuminate the matrix. A maximum slice thickness of about $2 \mathrm{~mm}$ could be investigated with the light intensity supplied by the $100 \mathrm{~W}$ halogen light bulb.

The micrographs were quantitatively analysed. The total number of fibres in the specimen cross-section was determined by automated image analysis using the UTHSCSA ImageTool program (developed at the University of Texas Health Science Center at San Antonio, Texas, and available on the Internet at http://ddsdx.uthscsa.edu/dig/). The number of 'black fibres' (see next section) was determined by manual counting.

\section{Results}

Figure 2 shows a typical transmitted light micrograph of an alumina fibre reinforced composite wire cross-section. The

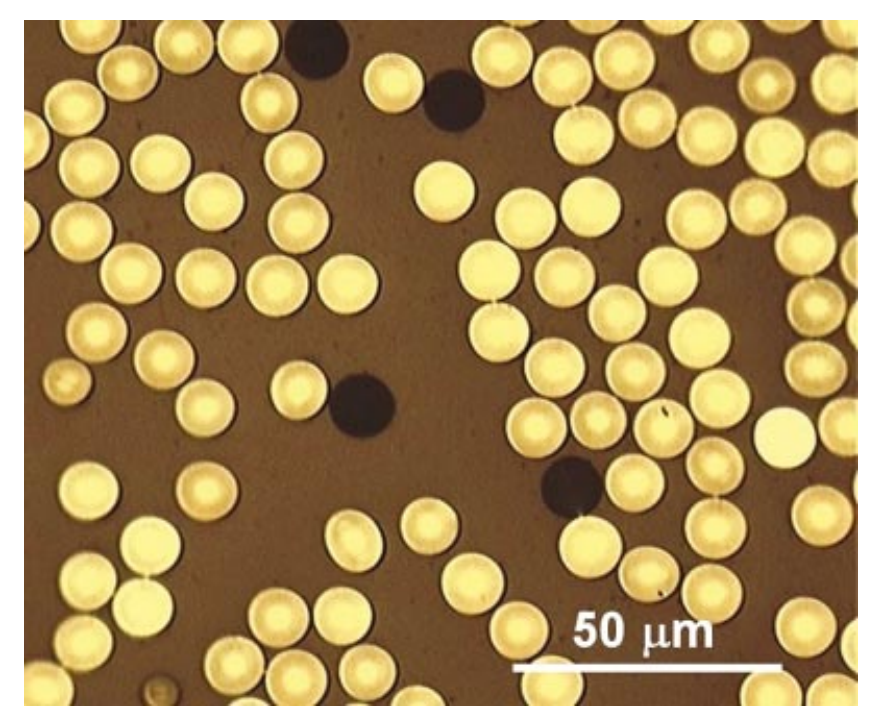

Fig. 2. Cross-section of a composite wire in transmitted light microscopy. The black spots are dead-ended fibres, whereas the bright spots are continuous fibres.

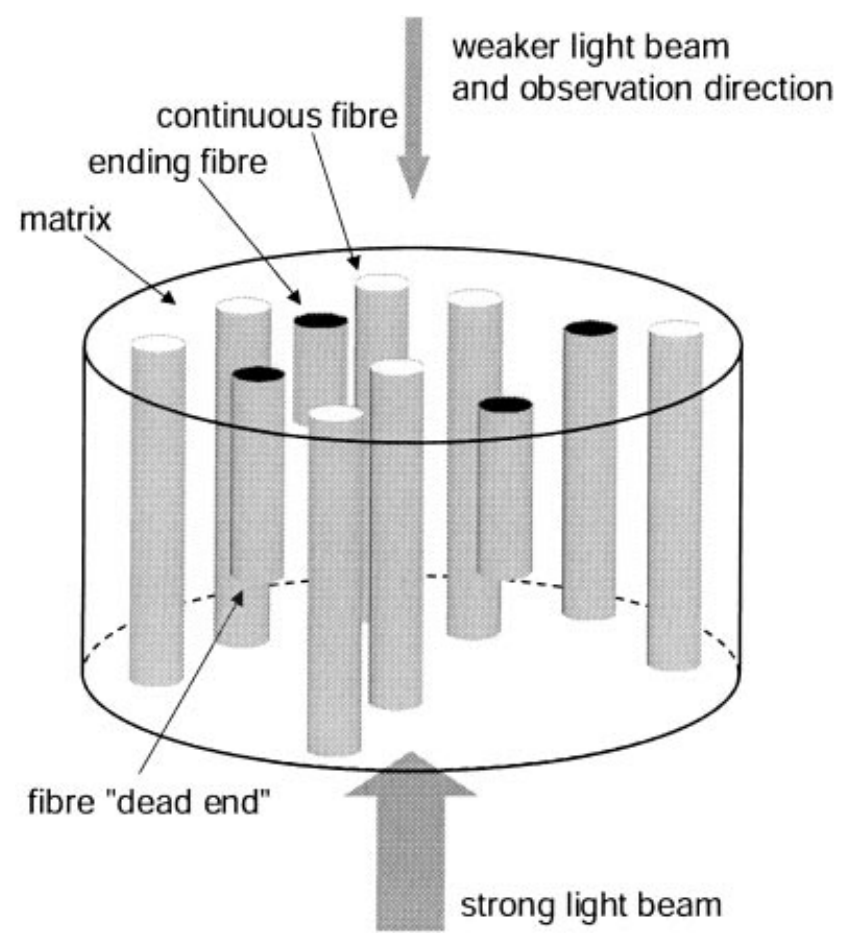

Fig. 3. Principle of the detection of dead-ended fibres in alumina fibre reinforced composites by the transmitted light microscopy technique.

fibres are clearly visible as bright spots (due to the transmitted light), whereas the matrix is slightly darker (the matrix would actually be completely black if no reflected light were used). The brightness of the fibres depends, among other parameters, on the light intensity and the slice thickness; however, other factors seem to intervene, as the brightness of individual fibres varies within the same metallographic sample (Fig. 2). 


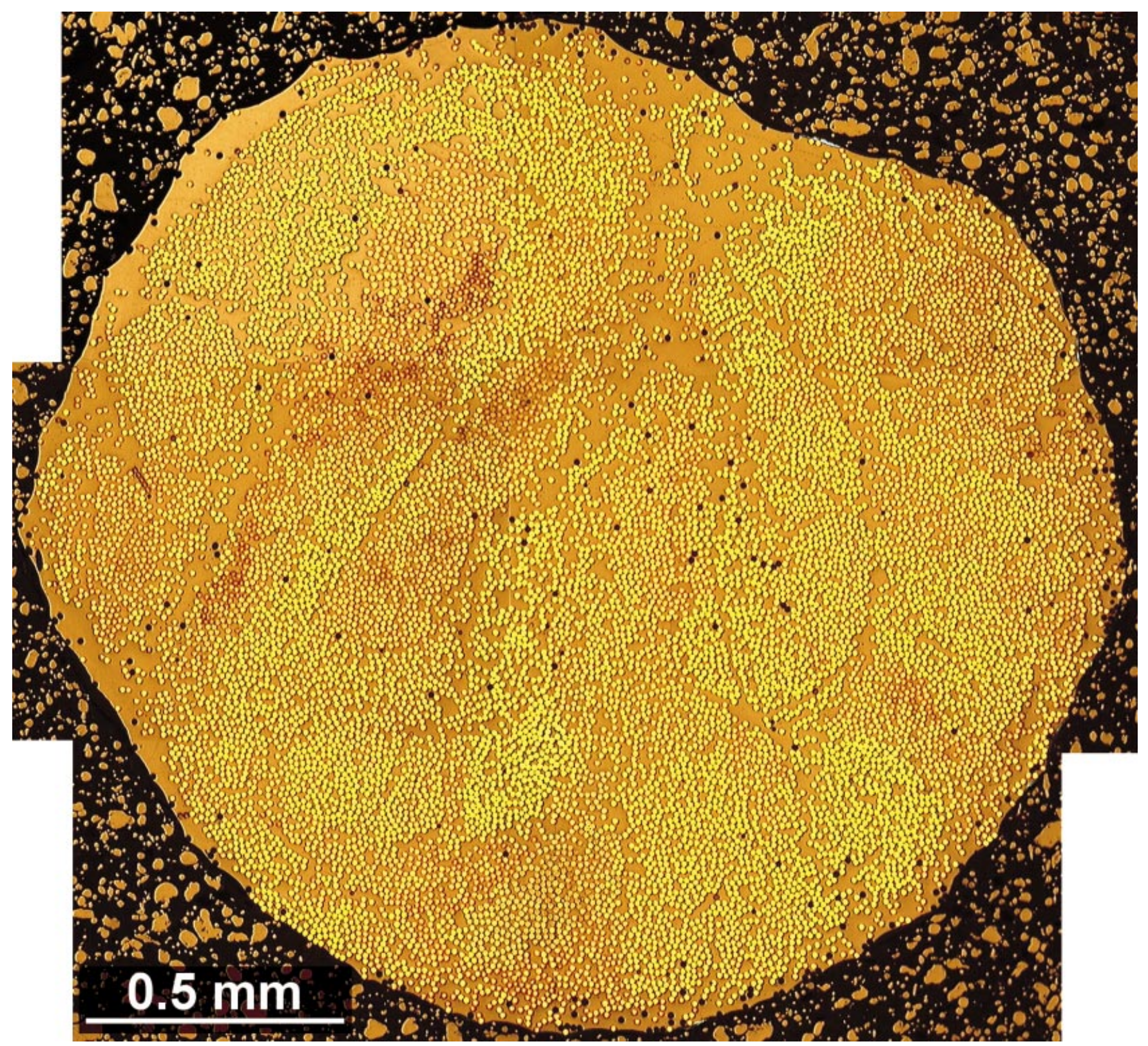

Fig. 4. Transmitted light micrograph of the entire cross-section of a virgin composite wire. The black spots are dead-ended fibres, the bright spots are continuous fibres and the rest is matrix.

Similar to the observation in reflected light microscopy, some fibres appear different in the transmitted light micrographs: instead of being brighter than the average, these fibres are completely black. Such fibres were identified, for the composite examined here, as dead-ended fibres, i.e. fibres that do not traverse the slice, as opposed to broken continuous fibres. This is illustrated schematically in Fig. 3. Two main reasons lead to this conclusion:

(i) Even on very thin slices a clearly identified black spot cannot be found in the same region, when the specimen is turned upside down, as one would expect for a symmetric defect such as a fibre break. (ii) The number of black spots does not change with prestressing of the composite, even though such prestressing was shown (using a different technique) to increase the number of broken fibres in this composite by a factor of up to 2 (Moser, 2002).

A significant advantage of the optical microscopy technique used here is that it allows for direct quantification of the density of the observed fibre defects because, unlike in reflected light microscopy, the observed material volume is known. Figure 4 shows a typical micrograph of the whole wire crosssection in the transmitted light mode. There are a considerable number of dead fibre ends visible in the form of black spots. 


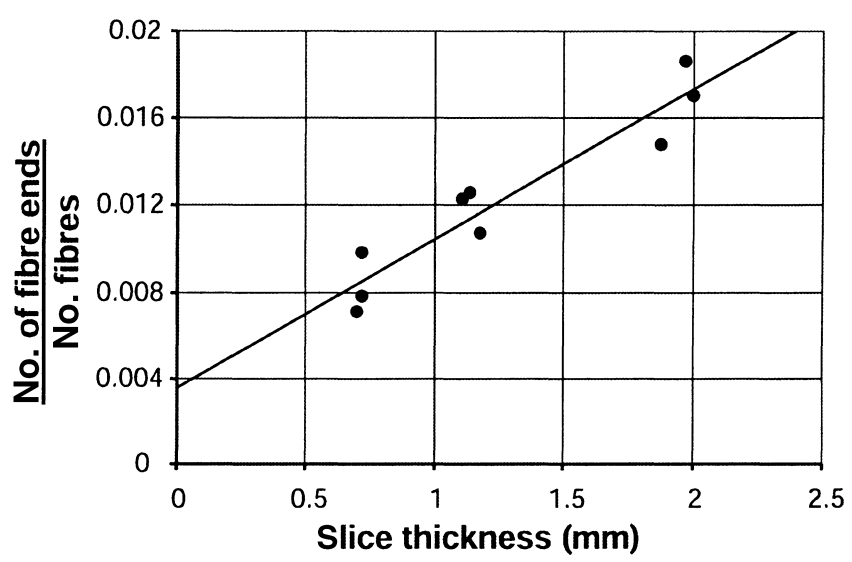

Fig. 5. Ratio of dead fibre ends and the total number of fibres in the crosssection as a function of slice thickness in a virgin composite wire.

The ratio of dead fibre ends to the total number of fibres in the cross-section is plotted in Fig. 5 for three different slice thicknesses (corresponding to three different volumes). The measurement indicates, as expected, a linear increase in the number of dead fibre ends with thickness.

It can be noted from this figure that there is an offset at a slice thickness of zero: this offset was found to be real and reproducible. Some of the black fibres may therefore be an artefact of the metallographic preparation technique. However, it is clear that the increase in the number of dark fibres that results from an increase in slice thickness corresponds to the detection of real defects, situated within the volume of the material.

A damage parameter, $D_{0}$, denoting the number of fibre ends per millimetre of fibre, can be defined as the slope of the curve in Fig. 5. This parameter takes a value of 0.0068 for the composite wire investigated in this study.

\section{Discussion and conclusions}

The nature of the damage found in the composite by the transmitted light microscopy method can be explained as a result of the processing of this composite wire. Either ending fibres in the initial fibre tow, or fibre fracture followed by relative displacement of the fractured fibre ends during wire processing prior to matrix infiltration and solidification, can lead to dead ending fibres in the final composite. The value of the damage parameter $D o$ was quantitatively verified using a different test method, based on tensile tests on the composite wire after melting the matrix (Moser, 2002).

Careful observation of the micrographs revealed, in agreement with the results of the mechanical tests, an inhomogeneous distribution of the defective fibres: the majority of these are situated either at the periphery of the wire, or at the border of essentially fibre-free matrix regions, which correspond to regions between individual fibre tows that were bundled and infiltrated when making the composite. These observations indicate that ending fibres in the wire originate mainly from surface damage caused to the fibre tows or to the liquid matrix composite wire during spooling and handling operations prior to matrix solidification.

Although sample polishing and preparation is somewhat more demanding than in conventional metallography, there are several advantages to the present technique. As the sample volume material is known, the density of damage can be quantified, something that cannot be done in reflected light microscopy of transverse composite cross-sections because the maximum depth distribution of identifiable defects is generally unknown. Compared with the use of longitudinal sections in reflected light microscopy (Nicholas et al., 1997), where such dead ends or also fibre breaks can indeed be observed, the present technique has the advantage that the sample volume is obviously larger (by roughly two orders of magnitude), and that it is more immune to polishing artefacts. This makes measurement and quantification of the fibre damage density significantly easier.

An interesting observation is the effect of fibre misalignment that is found in live transmitted light microscopy. As illustrated in Fig. 6, fibre misalignment can readily be seen because a misoriented fibre moves relative to its well-aligned neighbours when the focus plane of the microscope is altered.

A second observation worth noting is that the visual aspect of all fibres is not the same within a given composite sample: some fibres appear brighter than others, whereas some display a shaded outer ring of variable width and darkness (Figs 2 and 6). We have no firm explanation to offer for this observation; however, given the symmetric shape of the rings, we propose

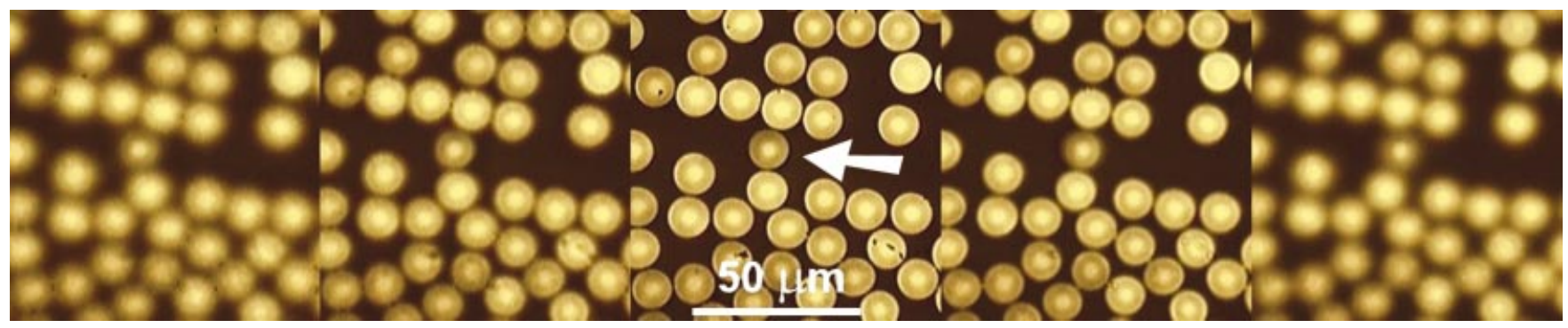

Fig. 6. A misaligned fibre (arrow) moves relative to its neighbours when the focus of the microscope is changed in transmitted light microscopy. 
that this effect may be caused by fibre-to-fibre variations in the reflectivity of light along the fibre-matrix interface. This could be due to, for example, variations in the fibre surface roughness.

In conclusion, we propose a simple method for the microscopic characterization of fibre reinforced composites with the following characteristics:

(i) the method can be used with any composite system where the fibres are transparent or translucent (e.g. glass or fine-grained ceramic fibre reinforced composites).

(ii) it allows for quantification of damage or defects that affect the transmission of light along the fibre length; in the present work we identify dead-ended fibres as such a defect in infiltrated aluminium-alumina composites.

Finally, it might be of interest to explore the use of light sources other than standard white light. Monochromatic light sources might allow the detection of broken continuous fibres where the broken ends are still close together.

\section{Acknowledgements}

This work was funded by a joint EMPA/EPFL PhD thesis program, and by internal funding within EMPA in Thun and the Laboratory for Mechanical Metallurgy at EPFL. The authors would like to thank the 3M Company for the donation of the composite wire used throughout this study.

\section{References}

3M Company (2002) Composite Conductor Flyer. http://www.mmm.com/ market/industrial/mmc/comp_cond.html

3M Company (2001) Nextel Ceramic Textiles Technical Notebook. http:// www.mmm.com/ceramics.

Bunsell, A.R. \& Berger, M.-H. (2000) Fine diameter ceramic fibres. J. Euro. Ceram. Soc. 20, 2249-2260.

McCullough, C., Mortensen, A., Werner, P.S., Dève, H.E. \& Anderson, T.L. (2001) Fiber reinforced aluminum matrix composite wire. United States Patent 6245 425. B1, 12 June 2001.

Moser, B. (2002) Deformation and fracture of continuous alumina fibre reinforced aluminium, Doctoral Thesis, Swiss Federal Institute of Technology, Lausanne (EPFL), Switzerland.

Nicholas, T., Castelli, M.G. \& Gambone, M.L. (1997) Fiber breakage in metal matrix composites - reality or artifact? Scripta Mater. 36/5, 585-592.

Wilson, D.M. \& Visser, L.R. (2001) High performance oxide fibers for metal and ceramic composites. Composites, 32A, 1143-1153. 